\title{
InAs Diodes Fabricated Using Be Ion Implantation
}

\author{
Benjamin S. White, Ian C. Sandall, John P. R. David, Fellow, IEEE, and Chee Hing Tan, Member, IEEE
}

\begin{abstract}
Be ion implantation and annealing conditions were optimized to demonstrate an effective method for selective area p-type doping in InAs. Optimized implantation and annealing conditions were subsequently utilized to produce planar InAs diodes. The Be implanted planar diodes had a superior dynamic resistance-area product and comparable dark current with n-i-p InAs mesa diodes when operated at low temperatures.
\end{abstract}

Index Terms-Annealing, indium arsenide, ion implantation, photodiode.

\section{INTRODUCTION}

$\mathbf{I}$ nAs is considered a key III-V semiconductor in the fields of high speed and optoelectronics due to its high electron mobility and large band offset at the InAs/AlSb heterojunction. In addition, InAs has been shown to exhibit very low excess avalanche noise [1] and has a peak photoresponse close to $3.4 \mu \mathrm{m}$, which is ideally suited for methane gas sensing. The majority of reported InAs devices are mesa structures grown by molecular beam epitaxy (MBE) or metal-organic vapor phase epitaxy (MOVPE). Despite the known advantages of planar structures, planar InAs devices are seldom reported. This fact stems from an underdevelopment in selective area doping techniques, such as diffusion or ion implantation, which are crucial for planar device fabrication. In the drive to develop planar InAs devices, Iwamura and Watanabe [2] reported on $\mathrm{Zn}$ diffusion techniques which have been utilized to produce planar InAs diodes. However, complications associated with the diffusion techniques make it desirable to explore other doping methods; for example, when diffusing $\mathrm{Zn}$ into InAs, the high temperature environment necessitates an As overpressure to be maintained in the chamber to prevent the dissociation of As from the InAs. Consequently, $\mathrm{Zn}$ diffusion into InAs has often been carried out in a MOVPE reactor, which significantly increases the cost and complexity of the process [2], [3]. Furthermore, a protective mask that matches the thermal expansion coefficient of InAs is not readily available, and so thermally mismatched masks are commonly used. Compared with diffusion techniques, ion implantation has the advantage

Manuscript received February 17, 2015; revised May 12, 2015 and June 17, 2015; accepted July 8, 2015. This work was supported in part by the European Space Agency under Grant 4000107110, in part by the Engineering and Physical Sciences Research Council under Grant EP/H031464/1, in part by a LAND CASE studentship, and in part by the Defense Science and Technology Laboratory under Grant DSTLX1000064084. The review of this paper was arranged by Editor J. R. Tower.

The authors are with the Department of Electronic and Electrical Engineering, University of Sheffield, Sheffield S1 3JD, U.K. (e-mail: ben.white@sheffield.ac.uk; i.sandall@sheffield.ac.uk; j.p.david@sheffield.ac.uk; c.h.tan@sheffield.ac.uk).

Color versions of one or more of the figures in this paper are available online at http://ieeexplore.iee.org.

Digital Object Identifier 10.1109/TED.2015.2456434 of being a low temperature process thereby avoiding the complications associated with the high temperature processing of InAs stated above; in addition, a large variety of dopants and more intricate doping profiles are achievable. Ion implantation of the light group IIa elements, $\mathrm{Be}$ and $\mathrm{Mg}$, has proved popular for p-type doping in a variety of III-V semiconductors such as InSb [4] and GaAs [5]. The popularity of these particular elements is owing to the low implant damage inflicted during the implant and the excellent damage removal through postimplant annealing, which can be a major drawback of doping III-V semiconductors using ion implantation [6]. Mg implantation into InAs was found to be ineffective for p-type doping, and it is speculated that the radiation defects cancelled out the usual acceptor like behavior of $\mathrm{Mg}$ resulting in a neutral net doping [7]. However, Wang et al. [8] reported on the damage accumulation and lattice strain of $\mathrm{Be}$ implanted InAs. Using an implant energy of $80 \mathrm{keV}$ and a dose up to $4 \times 10^{13} \mathrm{~cm}^{-2}$, creating a peak Be concentration of $1.1 \times 10^{18} \mathrm{~cm}^{-3}$, minimal lattice strain is introduced to the crystal and only point defects are formed after the implant. The Be profile before and after annealing of Be implanted InAs has been reported by Gerasimenko et al. [9], [10]. When comparing the Be profile against a simulation generated using software package Transport of Ions in Matter (TRIM) [11], errors in the straggle of up to $24 \%$ were found. In addition, the severe diffusion of Be when annealed at temperatures greater than $450{ }^{\circ} \mathrm{C}$ on time scales of 30 minutes was highlighted. Be implantation has not been utilized to produce InAs devices, nor the electrical properties of Be implanted InAs been tested.

The key contributions of this paper are: 1) Be implantation and annealing have been demonstrated as an effective method for selective area p-type doping InAs; 2) implant and annealing conditions have been optimized to maximize the electrical performance of InAs diodes; 3) secondary ion mass spectrometry (SIMS) measurements were used to observe the diffusion of Be caused by annealing; and 4) high-quality Be-implanted planar InAs diodes have been demonstrated with comparable dark currents to eptiaxially grown n-i-p mesa InAs diodes.

\section{EXPERIMENTAL Details}

Two InAs i-n structures were used in this paper. The first structure, hereafter, referred to as wafer A, was grown by MBE at approximately $490{ }^{\circ} \mathrm{C}$ and consisted of a $3.5 \mu \mathrm{m}$ intrinsic layer on a $2 \mu \mathrm{m} \mathrm{Si}$-doped $\mathrm{n}$ layer grown on a $\mathrm{n}^{+}$ InAs substrate. The second structure, wafer $\mathrm{B}$, was grown using MOVPE with a susceptor temperature of $590{ }^{\circ} \mathrm{C}$ and consisted of a $6 \mu \mathrm{m}$ thick intrinsic layer on a $2 \mu \mathrm{m} \mathrm{Si-doped}$ $\mathrm{n}$ layer grown on a $\mathrm{n}^{+}$InAs substrate. Be ion implantation was used to define a p-type doping profile in each of the structures. Wafer A was subject to a uniform blanket implant 
of $\mathrm{Be}$ and subsequently used to fabricate all of the mesa diodes presented in this paper. Wafer B was patterned with a dielectric mask before implantation and subsequently used to fabricate all of the planar diodes presented in this paper. TRIM was used to model the distribution of Be ions implanted into InAs. Simulations predicted that a flat doping profile to a depth of $0.75 \mu \mathrm{m}$, with the junction extending a total of $1 \mu \mathrm{m}$, could be realized from two ${ }_{9} \mathrm{Be}+$ implants with conditions of $200 \mathrm{keV}$ at $1 \times 10^{14} \mathrm{~cm}^{-2}$ and $70 \mathrm{keV}$ at $3.8 \times 10^{13} \mathrm{~cm}^{-2}$. The implantation was carried out at University of Surrey Ion Beam Center. A $1 \mu \mathrm{m} \mathrm{p}$ region was chosen to limit the damage to the crystal. The beam current was set to $20 \mu \mathrm{A}$ to avoid any localized heating of the wafers. The wafers were held $7^{\circ}$ off the beam axis toward the (100) crystal plane during the implant to avoid channeling effects and to enable comparisons against the TRIM model.

It has been reported that the implant temperature can have a pronounced effect on the resulting crystal quality within certain III-V semiconductors, such as InP [12], however, no such information exists for InAs. To investigate the effects of such dynamic annealing in InAs, implants were carried out on samples held at room temperature (RT), $100{ }^{\circ} \mathrm{C}$ and $200{ }^{\circ} \mathrm{C}$. After implantation, the samples were annealed at temperatures ranging from $450{ }^{\circ} \mathrm{C}$ to $700{ }^{\circ} \mathrm{C}$ for $30 \mathrm{~s}$ in a nitrogen rich atmosphere. Gerasimenko et al. [10] showed that significant Be diffusion occurs at temperatures as low as $400{ }^{\circ} \mathrm{C}$, and therefore to minimize the Be diffusion, rapid thermal annealing (RTA) was utilized. Precautions were taken to preserve the stoichiometry at the surface of the semiconductor during annealing. After annealing, and in the case of wafer A, circular mesa diodes of varying diameters were fabricated using a typical photolithographic and wet chemical etching technique [13]. Ti/Au was deposited as top and back contacts. The removal of radiation damage, which is inherently connected to the implant process, was analyzed through comparisons of the dark current of the mesa diodes. Appropriate annealing conditions were evaluated along with a discussion of the influence of substrate temperature during implantation. Optimized implant and annealing conditions were subsequently employed to produce planar diodes from wafer B. After annealing, the planar diodes had metal contacts deposited. Current-voltage measurements were performed using an Agilent B5100 parameter analyzer by probing the diodes.

\section{RESULTS AND DISCUSSION}

The current density-voltage $(J-V)$ characteristics of diodes from wafer A, implanted at RT and annealed at temperatures from $450{ }^{\circ} \mathrm{C}$ to $700{ }^{\circ} \mathrm{C}$ for $30 \mathrm{~s}$ are shown in Fig. 1. An unannealed diode from wafer $\mathrm{A}$ is also shown. In comparing the $J-V$ characteristic of an unannealed diode against one annealed at $450{ }^{\circ} \mathrm{C}$, no improvement is observed. It is difficult to identify whether the slight rectifying behavior observed in both diodes was due to Be activation, or if a Schottky junction at the metal semiconductor interface was the cause. However, in any case, the similarity between $J-V$ characteristics of unannealed diodes and those annealed at $450{ }^{\circ} \mathrm{C}$ suggests that significant $\mathrm{Be}$ activation and crystal recovery has not

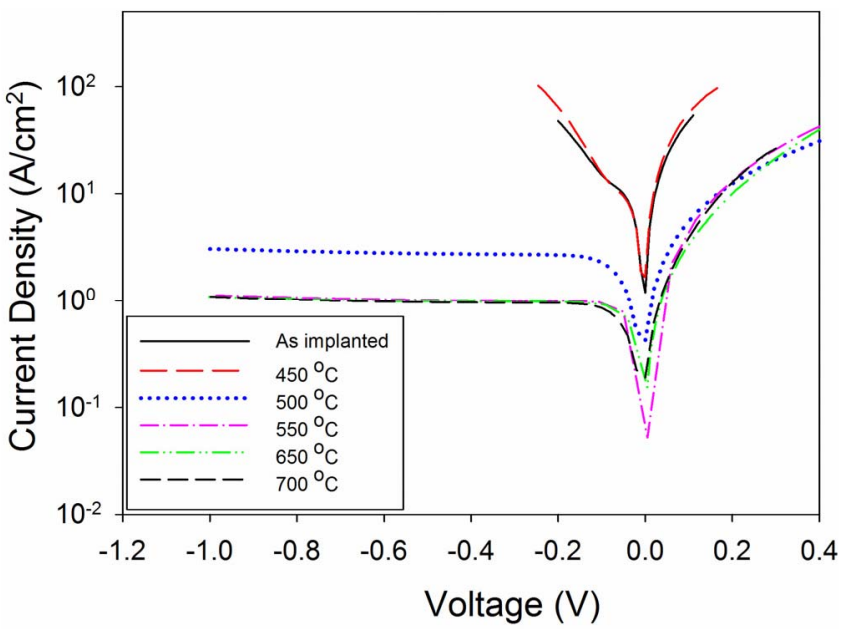

Fig. 1. $J-V$ characteristics of mesa InAs diodes implanted at RT and annealed at $450{ }^{\circ} \mathrm{C}, 500{ }^{\circ} \mathrm{C}, 550{ }^{\circ} \mathrm{C}, 650{ }^{\circ} \mathrm{C}$, and $700{ }^{\circ} \mathrm{C}$ for $30 \mathrm{~s}$. In addition, the $J-V$ characteristics an unannealed diode implanted at RT is shown.

occurred. Diodes annealed at $500{ }^{\circ} \mathrm{C}$ displayed a pronounced rectifying behavior, however, the lowest dark current density was measured in diodes that had been annealed at temperatures of $550{ }^{\circ} \mathrm{C}-700{ }^{\circ} \mathrm{C}$. Diodes annealed in this temperature range produced very similar $J-V$ characteristics with a dark current density of $1 \mathrm{Acm}^{-2}$ and a forward $J-V$ characteristic that was limited by the series resistance of the semiconductormetal interface. It is apparent that the maximum recovery is achieved from annealing at a temperature of $550{ }^{\circ} \mathrm{C}$, and higher annealing temperatures do not encourage further levels of recovery to reduce the dark current density of the resulting diodes. However, this does not necessarily indicate that the annealing has fully eradicated all of the implant damage; often in III-V semiconductors there is residual implant damage, which is immune to dissociation through annealing.

Hot implants have been shown to mitigate the implant damage leading to higher quality crystal after annealing. The $J-V$ characteristics of diodes implanted at RT, $100{ }^{\circ} \mathrm{C}$, and $200{ }^{\circ} \mathrm{C}$ are shown in Fig. 2. In addition to this, samples implanted at $\mathrm{RT}, 100{ }^{\circ} \mathrm{C}$, and $200{ }^{\circ} \mathrm{C}$ and annealed at $500{ }^{\circ} \mathrm{C}$ and $550{ }^{\circ} \mathrm{C}$ are also shown. The annealing temperature clearly segregates the $J-V$ characteristics of the diodes into three distinct bands. Within each band, the $J-V$ characteristics of diodes from the hot implants closely resemble those from the RT implant study. Therefore, the implant temperature is seen to have a negligible effect on the resulting device $J-V$ characteristics. This result is similar to that obtained in various other studies, such as Be implantation into InP [14].

Our dark current characteristics confirm that Be has been successfully implanted and activated. We now focus our attention to the Be profile within our samples. Fig. 3 shows Be profiles before and after postimplant annealing as measured by SIMS. The Be profile generated using TRIM is included for comparison. Discrepancies seen in the absolute value of the Be concentration in the TRIM and SIMS results are likely to be caused by a calibration error of the SIMS measurement. Unannealed samples at RT and $200{ }^{\circ} \mathrm{C}$ showed 


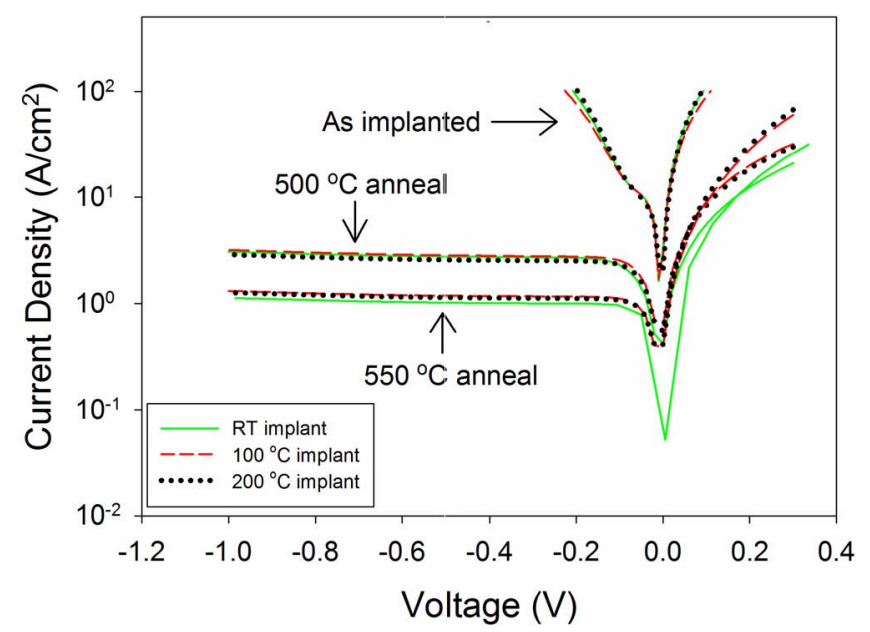

Fig. 2. $J-V$ characteristics of mesa InAs diodes which were implanted at RT, $100{ }^{\circ} \mathrm{C}$, and $200{ }^{\circ} \mathrm{C}$. In addition, the $J-V$ characteristics of diodes annealed at $500{ }^{\circ} \mathrm{C}$ and $550{ }^{\circ} \mathrm{C}$ for $30 \mathrm{~s}$ from the three implant temperatures are also shown.

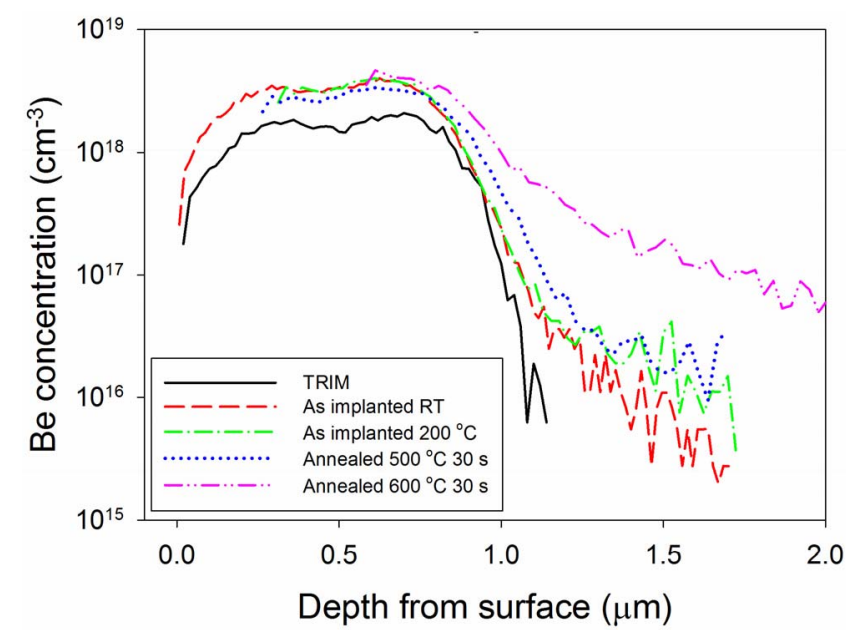

Fig. 3. The concentration of Be after implantation into InAs at: room temperature; $200{ }^{\circ} \mathrm{C}$; room temperature and annealed at $500{ }^{\circ} \mathrm{C}$ for $30 \mathrm{~s}$; and room temperature and annealed at $600{ }^{\circ} \mathrm{C}$ for $30 \mathrm{~s}$. In addition, a simulated Be profile generated using TRIM is shown.

identical Be distributions down approximately $3 \times 10^{16} \mathrm{~cm}^{-3}$, showing that the substrate temperature does not affect the resulting Be profile. The 70 and $200 \mathrm{keV}$ implants produced Be concentration peaks at 0.29 and $0.64 \mu \mathrm{m}$, respectively. When the tail of the $200 \mathrm{keV}$ implant is compared against the TRIM model, the SIMS results showed that the projected range was slightly shorter and the straggle was greater then simulated. Results are summarized in Table I. Previous work reported errors of up to $24 \%$ in the straggle but the projected range was found to be accurate [9].

Our comparison showed TRIM to be in much better agreement with experimental data. However, the discrepancy between studies may be due to increased ion interactions with defects in higher doses. Samples implanted at RT and annealed at $500{ }^{\circ} \mathrm{C}$ and $600{ }^{\circ} \mathrm{C}$ for $30 \mathrm{~s}$ were included in the SIMS analysis. A slight redistribution of $\mathrm{Be}$ was observed when annealing at $500{ }^{\circ} \mathrm{C}$ as the peak concentration of $\mathrm{Be}$ had reduced by $16 \%$ and the Be tail extending into the sample had broadened.
TABLE I

Summarized Results Analyzing the TaIl of Be Distribution FROM TRIM AND SIMS MEASUREMENTS

\begin{tabular}{cccc}
\hline \hline Sample & $\begin{array}{c}\text { Projected } \\
\text { Range } \\
(\mathrm{nm})\end{array}$ & $\begin{array}{c}\text { Straggle } \\
(\mathrm{nm})\end{array}$ & $\begin{array}{c}\text { Peak Concentration } \\
\left(\mathrm{cm}^{-3}\right)\end{array}$ \\
\hline TRIM & 700 & 130 & $2.0 \times 10^{18}$ \\
$\begin{array}{c}\text { SIMS } \\
\left(\begin{array}{l}\text { Unannealed, } \\
\text { implanted at } R T \\
\left.\text { and } 200^{\circ} \mathrm{C}\right)\end{array}\right.\end{array}$ & 645 & 147 & $4.0 \times 10^{18}$ \\
$\begin{array}{c}\text { SIMS } \\
\text { (Implanted at } R T, \\
\text { annealed at } 500 \\
\left.{ }^{\circ} \mathrm{C} \text { for } 30 \mathrm{~s}\right)\end{array}$ & - & 175 & $3.4 \times 10^{18}$ \\
\hline \hline
\end{tabular}

The Be profile of the tail after the anneal has been modeled by a Gaussian distribution with the results summarized in Table I. The redistribution of Be resulting from a $600{ }^{\circ} \mathrm{C}$ anneal for $30 \mathrm{~s}$ was much more severe, resulting in a distorted Be profile that could not be modeled by a Gaussian distribution. The peak Be concentration in the sample annealed at $600{ }^{\circ} \mathrm{C}$ for $30 \mathrm{~s}$ was found to be slightly larger than the unannealed samples, suggesting another mechanism other than diffusion may be occurring. A similar observation was reported for $\mathrm{Mg}$ implants into InAs [7]; the explanation offered was that, during annealing, the highly mobile $\mathrm{Mg}$ tends to gather around radiation defects forming $\mathrm{Mg}$ clusters. It is possible that $\mathrm{Be}$ behaves in a similar manner to $\mathrm{Mg}$ during high temperature annealing. Annealing at $550{ }^{\circ} \mathrm{C}$ appears to be the optimum annealing condition to achieve low dark currents and maintain the implanted Be profile.

Having analyzed the effects of implant temperature and postimplant annealing, we used our results to fabricate planar diodes on a sample from wafer B. Planar diodes of varying diameters were fabricated using RT ion implantation and annealing conditions of $550{ }^{\circ} \mathrm{C}$ for $30 \mathrm{~s}$. The $J-V$ characteristics of the resulting diodes are shown in Fig. 4. Strong similarities exist between the $J-V$ characteristics of the planar diodes, and the mesa diodes shown in Fig. 1, all having a dark current density of $1 \mathrm{Acm}^{-2}$, which remains relatively flat through the voltage range measured. The planar diodes showed bulk dominated dark current and good confinement of the depletion region as shown in Fig. 4 by scaling the dark current of various sized diodes with their respective active areas. Such strong correlation between the dark current density of various sized diodes could only be attained if the p-n junction was formed at the boundary of the p-type implanted region. These features are desirable for the development of closely spaced InAs photodiode arrays.

The RT and $200 \mathrm{~K} J-V$ characteristics of a $\mathrm{Zn}$ diffused planar diode from Iwamura and Watanabe [2], a MOVPE grown mesa diode from Ker et al. [15] and a commercial photodiode from Judson are also plotted in Fig. 4. At $-0.1 \mathrm{~V}$, the dark current density of the Be implanted diodes were found to be larger than that of the reference diodes when measured at RT. However, at $200 \mathrm{~K}$, the dark current density of the Be implanted diode has reduced by over three orders 


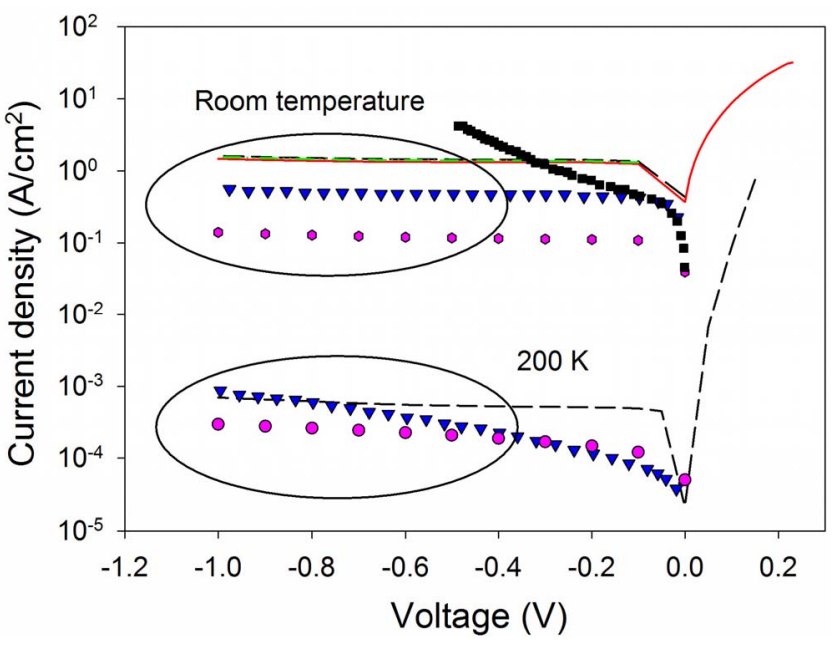

Fig. 4. $J-V$ characteristics of 100,200 , and $300 \mu$ m diameter Be implanted planar InAs diodes at RT are shown by a dashed and dotted green line, solid red line, and dashed black line, respectively. The $J-V$ characteristic of a $300-\mu \mathrm{m}$ diameter Be implanted planar InAs diode is also shown at $200 \mathrm{~K}$ by a dashed black line. The $J-V$ characteristics of a $\mathrm{Zn}$ diffused planar diode [2] (inverted blue triangles), an MOVPE grown mesa diode [15] (pink circles), and a commercial photodiode from Judson (black squares) are shown at RT and $200 \mathrm{~K}$.

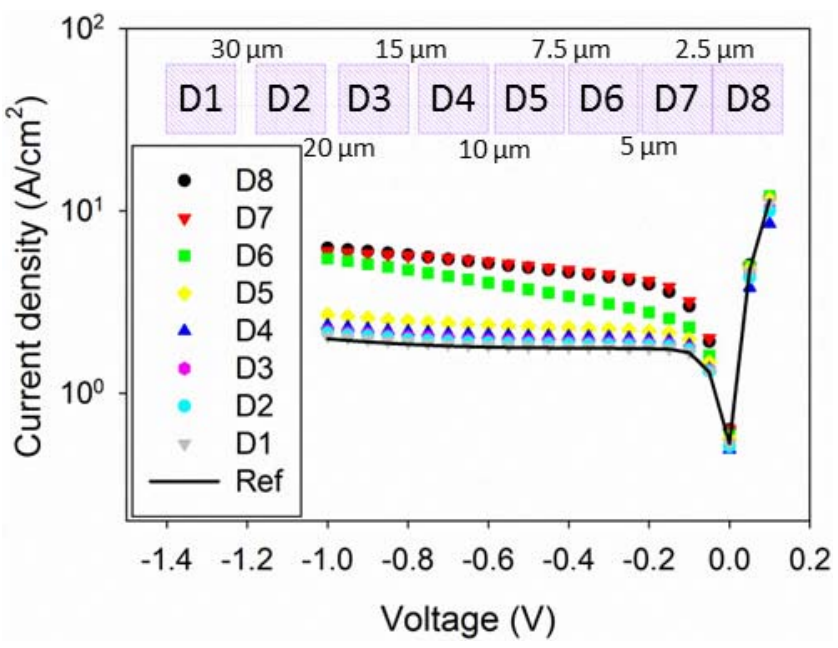

Fig. 5. Schematic diagram of the linear array of $100 \mu \mathrm{m}$ square planar InAs diodes fabricated with the various separation distances (insert). The $J-V$ characteristics of the diodes from the linear array are shown in addition to the $J-V$ characteristics of an isolated reference planar diode.

of magnitude. The dark current density of the $\mathrm{Zn}$ diffused diode and the mesa diode had also reduced by a similar amount at this temperature. However, the dark current density was found to increase significantly with reverse bias, whereas the dark current density of the Be implanted diode remained relatively constant across the voltage range. Owing to this fact, the maximum dynamic resistance-area product $\left(R_{d} \mathrm{~A}\right)$ of the Be implanted diodes was measured to be $12.1 \mathrm{k} \Omega \cdot \mathrm{cm}^{-2}$ at $-0.3 \mathrm{~V}$ which is larger than that of a $\mathrm{Zn}$ diffused planar diode [2] or n-i-p mesa diode [15] with $R_{d}$ As of 2.9 and $6 \mathrm{k} \Omega \cdot \mathrm{cm}^{-2}$ at -0.1 and $-0.75 \mathrm{~V}$, respectively.

Be implantation and annealing techniques discussed in this paper could be used to fabricate an array of InAs photodiodes for imaging applications. It is, therefore, useful to consider the performance of the diodes when in an array geometry. As a preliminary study, a linear array of $100-\mu \mathrm{m}$ square diodes was fabricated with various separation distances between neighboring diodes. The $J-V$ characteristic of each diode in the linear array is shown in Fig. 5 along with the $J-V$ characteristic from a reference of $100-\mu \mathrm{m}$ diameter planar diode. The reference diode was located over $120-\mu \mathrm{m}$ away from any other diode and can be considered as decoupled from any other device. A schematic of the linear array is shown in the inset of Fig. 5. Diodes D6, D7, and D8 have higher dark currents than the reference diode indicating incomplete isolation when the gap between diodes is $<5 \mu \mathrm{m}$. For diode separation above $7.5 \mu \mathrm{m}$, the dark currents from D5 to D1 were found to asymptotically approach the dark current of the reference diode. The zero bias dark current of diodes D5-D1 is within $\pm 6 \%$ of the reference diode. Further optimization of implantation and annealing conditions, use of additional implantation to increase the resistivity of InAs or use of isolation trenches will be required to reduce the separation distance.

The optical properties of the planar diodes are now investigated. The responsivity of a planar diode was measured at $1550 \mathrm{~nm}$ using a HeNe laser with the optical spot focused on the p-type region of the diode. The photocurrent was measured at room temperature using phase sensitive detection and found to be $0.45 \mathrm{~A} / \mathrm{W}$ without any antireflection (AR) coating. Further work to increase the minority carrier diffusion length and the use of an AR coating could improve this figure.

\section{CONCLUSION}

Be implantation has been demonstrated as an effective method for selective area p-type doping InAs. The implant process appears to be destructive, typically resulting in poor electrical characteristics of unannealed material, however, good recovery can be achieved using RTA. Annealing at a temperature of $550{ }^{\circ} \mathrm{C}$ provided the optimum conditions in terms of producing the lowest dark current in mesa diodes and preserving the as-implanted Be profile. Higher annealing temperatures did not produce better crystal quality and only caused further Be diffusion. Implants carried out at RT were found to be just as effective as hot implants. Planar InAs diodes were demonstrated by utilizing the optimized Be implantation techniques discussed in this paper. The dark current density of the Be implanted planar diodes was found to be larger than that of an InAs $\mathrm{Zn}$ diffused planar diode and mesa diode when measured at RT. However, at $200 \mathrm{~K}$, the $J-V$ characteristics were much more comparable, and the maximum $R_{d} \mathrm{~A}$ of the Be implanted diode was found to be larger than that of the $\mathrm{Zn}$ diffused planar diode and mesa diode. The lateral junction confinement of the planar diodes was investigated in an array geometry and a diode separation of $7.5 \mu \mathrm{m}$ was found to electrically isolate neighboring diodes. The responsivity of the planar diodes was found to be $0.45 \mathrm{~A} / \mathrm{W}$ at $1550 \mathrm{~nm}$.

\section{REFERENCES}

[1] A. R. J. Marshall, C. H. Tan, M. J. Steer, and J. P. R. David, "Extremely low excess noise in InAs electron avalanche photodiodes," IEEE Photon. Technol. Lett., vol. 21, no. 13, pp. 866-868, Jul. 1, 2009.

[2] Y. Iwamura and N. Watanabe, "InAs planar diode fabricated by Zn diffusion,' Jpn. J. Appl. Phys., vol. 39, no. 1, pp. 5740-5745, 2000. 
[3] A. Säynätjoki et al., "InAs pixel matrix detectors fabricated by diffusion of $\mathrm{Zn}$ in a metal-organic vapour-phase epitaxy reactor," Nucl. Instrum. Methods Phys. Res., vol. 563, no. 1, pp. 24-26, Jul. 2006.

[4] C. E. Hurwitz and J. P. Donnelly, "Planar InSb photodiodes fabricated by Be and $\mathrm{Mg}$ ion implantation," Solid-State Electron., vol. 18, no. 9, pp. 753-756, Sep. 1975.

[5] R. G. Hunsperger, "Mg and Be ion implanted GaAs," J. Appl. Phys., vol. 43, no. 3, p. 1318 , Nov. 1972.

[6] W. Wesch, "Ion implantation in III-V compounds," Nucl. Instrum. Methods Phys. Res. B, vol. 68, nos. 1-4, pp. 342-354, May 1992.

[7] N. N. Gerasimenko, A. M. Myasnikov, V. I. Obodnikov, and L. N. Safronov, "Redistribution of magnesium in InAs during post-implantation annealing," Nucl. Instrum. Methods Phys. Res., vols. 80-81, pp. 924-926, 1993.

[8] Q. W. Wang et al., "Lattice expansion and evolution of damage buildup in Be-implanted InAs," Nucl. Instrum. Methods Phys. Res. B, vol. 269, no. 21, pp. 2527-2531, Nov. 2011.

[9] N. N. Gerasimenko, G. L. Kuryshev, A. M. Myasnikov, V. I. Obodnikov, and I. V. Verner, "Distribution of impurities implanted in InSb and InAs before and after annealing," Nucl. Instrum. Methods Phys. Res. B, vols. 127-128, pp. 446-450, May 1997.

[10] N. N. Gerasimenko, G. S. Khryashchev, G. L. Kuryshev, A. M. Myasnikov, and V. I. Obodnikov, "SIMS study on redistribution of implanted impurities in InSb and InAs during post-implantation annealing," Nucl. Instrum. Methods Phys. Res. B, vol. 111, nos. 3-4, pp. 281-284, 1996.
[11] J. P. Biersack and L. G. Haggmark, "A Monte Carlo computer program for the transport of energetic ions in amorphous targets," Nucl. Instrum. Methods, vol. 174, nos. 1-2, pp. 257-269, Aug. 1980.

[12] E. Wendler, T. Opfermann, and P. I. Gaiduk, "Ion mass and temperature dependence of damage production in ion implanted InP," J. Appl. Phys., vol. 82, no. 12 , p. 5965-5975, 1997.

[13] A. R. J. Marshall, C. H. Tan, J. P. R. David, J. S. Ng, and M. Hopkinson, "Fabrication of InAs photodiodes with reduced surface leakage current," Proc. SPIE, vol. 6740, p. 67400H, Oct. 2007

[14] J. P. Donnelly and C. E. Hurwitz, "The effect of implant temperature on the electrical characteristics of ion implanted indium phosphide," Solid-State Electron., vol. 23, no. 9, pp. 943-948, Sep. 1980.

[15] P. J. Ker, J. P. R. David, and C. H. Tan, "Temperature dependence of gain and excess noise in InAs electron avalanche photodiodes,' Opt. Exp., vol. 20, no. 28, p. 29568, Dec. 2012.

Authors' photographs and biographies not available at the time of publication. 